\title{
A SIMULATION ANALYSIS OF A PATIENT-CENTERED SURGICAL HOME TO IMPROVE OUTPATIENT SURGICAL PROCESSES OF CARE AND OUTCOMES
}

\author{
Douglas Morrice \\ Dongyang (Ester) Wang \\ McCombs School of Business \\ Information, Risk, and Operations Management \\ The University of Texas at Austin \\ Austin, TX 78712, USA
}

\author{
Jonathan Bard \\ Cockrell School of Engineering \\ Operations Research \& Industrial Engineering \\ The University of Texas at Austin \\ Austin, TX 78712, USA
}

\author{
Luci Leykum \\ Susan Noorily \\ Poornachand Veerapaneni \\ School of Medicine \\ The University of Texas Health Science Center, San Antonio \\ San Antonio, TX 78229, USA
}

\begin{abstract}
The process of preparing patients for outpatient surgery is information intensive. However, medical records are often fragmented among different providers and systems. As a result, the preoperative assessment process is frequently prolonged by missing information, potentially leading to surgery delay or cancellation. In this study, we simulate an anesthesiology pre-operative assessment clinic to quantify the impact of patient information deficiency and to assist in the development of a patient-centered surgical home to mitigate this problem through better system-wide coordination.
\end{abstract}

\section{INTRODUCTION}

In recognition of the need for better coordination of care, the American Society of Anesthesiologists has conceptualized the "Peri-operative" or "Patient-Centered Surgical Home (PCSH)" (ASA 2011). Under the PCSH model, care is integrated and coordinated among specialties, with Anesthesiologists and General Internists / Hospitalists designing or leading these integrated processes in collaboration with their colleagues. While this approach has conceptual appeal, it has not yet been widely implemented or studied.

In this project, our goal is to develop and implement a PCSH model in the outpatient surgery process at the University of Texas Health Science Center - San Antonio (UTHSCSA) and University Hospital, the acute care facility for University Health System (UHS), UTHSCSA's primary teaching affiliate. More specifically, we seek to improve surgical patient outcomes by redesigning pre- and peri-operative processes of care and to create a unified pathway of care that is treated like a single episode with tightly interconnected providers. The current system suffers from lack of coordination, fragmented surgical episodes of care, inefficiencies in the processes, patient information deficiencies, system congestion, and surgery delays and cancellations. These issues lead to significant problems across the system. Additionally, high utilization of the operating rooms and inpatient beds make addressing these issues critically important, particularly during a time of strategic growth in many service line areas at UHS. 
Toward our goal, we conducted a systems-level process analysis and a more detailed process analysis for the Anesthesia Preoperative Clinic (APC). The APC is the key clinic for system-wide coordination in outpatient surgery and the PCSH model. The detailed process analysis included a simulation study. The study revealed bottlenecks in APC, sources of process variation, and opportunities for process improvement. In addition, based on our analysis, APC was given the go-ahead by top UHS hospital management to implement the PCSH model.

The rest of the paper is organized in the following manner. Section 2 provides an overview of the system. Section 3 contains a process mapping and analysis of APC. In Section 4, we describe the APC simulation model and provide a validation of this model. Section 5 contains a simulation analysis of a APCcoordinated PSCH model. We conclude with a discussion of future work in Section 6.

\section{OUTPATIENT SURGERY}

Figure 1 contains a systems overview of the outpatient surgery process. Patients enter the system by visiting one of the eighteen UTHSCSA referral clinics. Those requiring surgery are scheduled for outpatient surgery at UHS. In the current system, about forty percent of the patients (usually those with more complicated medical conditions) are referred to the APC for a pre-operative assessment prior to their day of surgery to make sure that they are fit for the procedure. The remaining patients undergo an assessment on the day of surgery. This system-level analysis revealed a few important things:

1. There exists lack of coordination between the surgery clinics and APC.

2. APC can expand its role in the system by screening all patients prior to the day of surgery, thereby serving as "system coordinator" and heart of the PCSH model.

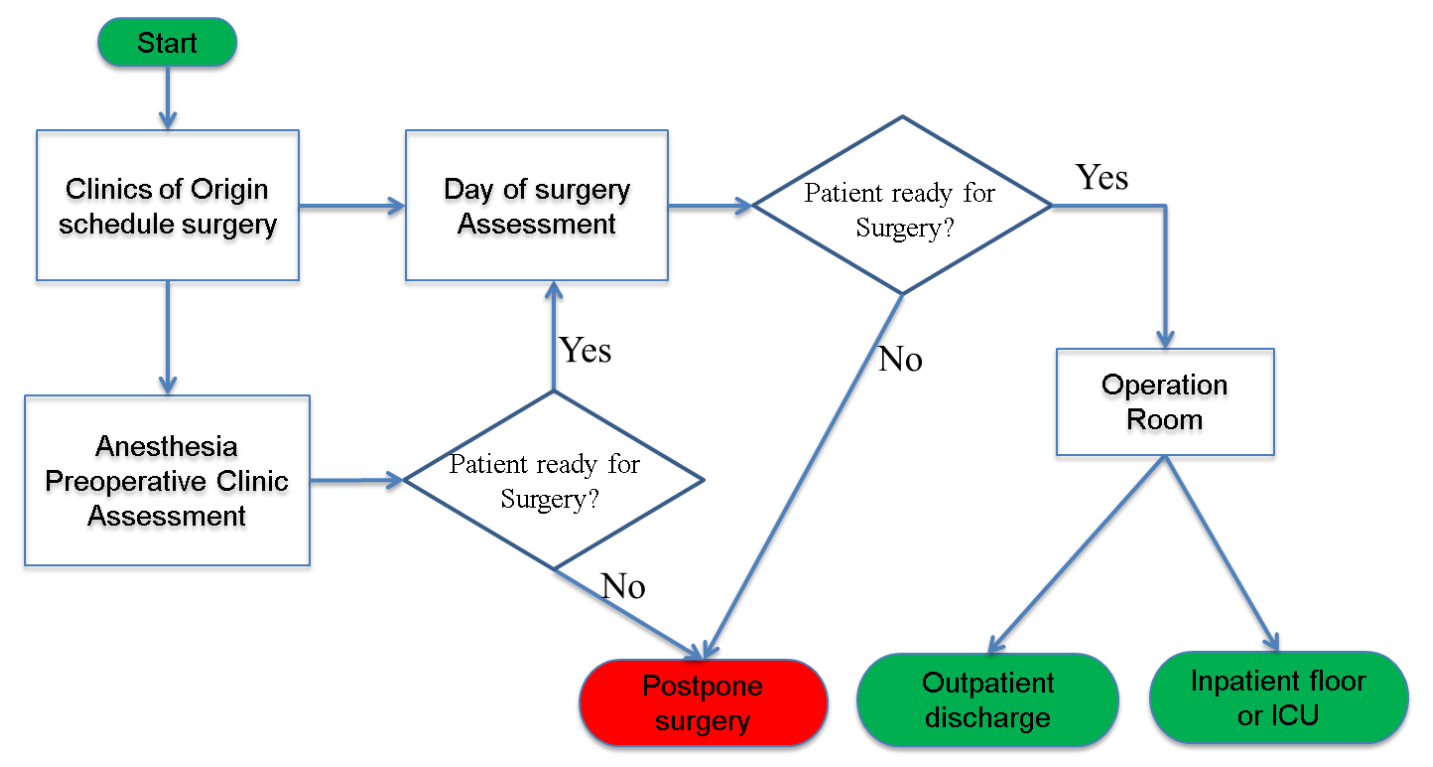

Figure 1: A system-level flowchart of the outpatient surgery process.

\section{ANESTHESIA PREOPERATIVE CLINIC}

\subsection{APC Process Mapping}

Due to APC's important role in this system, the second stage of our analysis focused on APC. Figure 2 contains a patient flow process mapping. The main steps include patient check-in and registration, a visit to the nurse for vitals and other information, provider examination with faculty oversight, provider information gathering, and patient discharge. Regarding resources, APC has one clerk who handles check-in 
and registration, one nurse, three providers (two residents and an Nurse Practitioner), and one attending Anesthesiologist (UTHSCSA faculty member). Note: "Pre-Op," "Pt," "RN," and "NP" stands for "preoperative," "patient," "Registered Nurse," and "Nurse Practitioner," respectively.

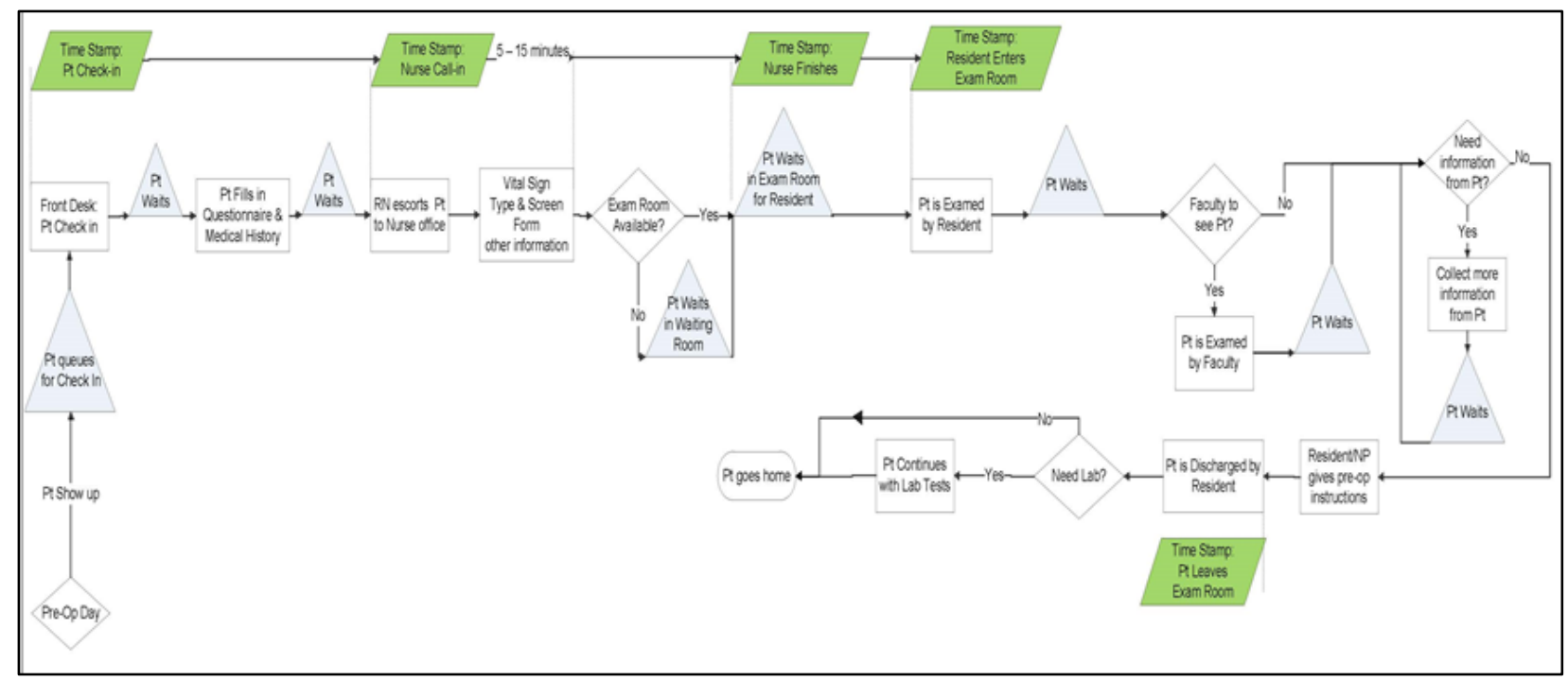

Figure 2: APC patient flow process mapping.

\subsection{APC Process Analysis}

We conducted an observational study of the APC process during a five week time period (June 28, 2012 to August 1,2012) that included 356 patients. When combined with historical data from clinic scheduling and patient check-in records, clinic referral information, and monthly patient visits, an analysis of these data revealed several things. First, providers were the limiting resource at APC (process bottleneck). More specifically, each provider spent, on average, 39 minutes with each patient and roughly another 1518 minutes (on average) attending to informational issues immediately after each patient's departure (total: 54-57 minutes). Since there were 3 providers, the process capacity was one patient every 18-19 minutes. Patients arrived at roughly one patient every 20 to 23 minutes. Therefore, provider utilization was in the 80 to $95 \%$ range. As a result, the APC process was running close to capacity, and experiencing high congestion, especially at certain times during the day (usually mid-morning).

Second, there was high variability in patient arrivals. We found substantial patient arrival rate variation both within and across days. Additionally, historical data showed a $15.45 \%$ average no-show rate and significant deviation between actual and scheduled arrival times.

We also found high variability in the amount of information that needed to be collected by providers for patients during an APC visit. This had significant impact on the provider assessment time. Each patient observed in the five week study was rated on level of information deficiency (low, medium, or high). A low rated patient required only straightforward updating of his/her electronic medical record (EMR). If, in addition, a patient required information from an external source (e.g., primary care provider or specialist) that required one phone call and some EMR searching, then this patient was considered medium. A high rated patient needed information from multiple external sources requiring more than one phone call and extensive searching of EMR databases. Figure 3 provides a summary of these results along with the impact of information deficiency on average provider assessment time. Fifty-nine percent of APC patients have some level of information deficiency. Average provider assessment time nearly doubles from low to high data deficiency. This is an example of the so-called "information hangover" discussed by Lahiri and Seidmann (2012). 
Lastly, demand for APC services was growing along a steady trend (see monthly demand for APC service in Figure 4). While the data contains significant seasonality, the trend of increasing demand is clear. In fact, the two highest months of demand ever encountered by APC were August and October 2012. During both of these months, we observed longer patient waiting times and clinic operating hours because the clinic was running very close to capacity (between 90 and $95 \%$ provider (bottleneck) utilization with highly variable arrival and service times).

\section{Patient Segmentation by Information Deficiency}

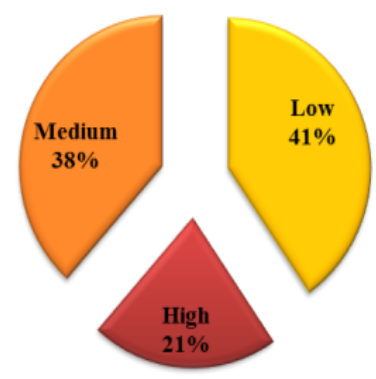

High: requires intensive data collecting work Low: complete or near complete medical record

\section{Provider Assessment Time}

ASA 3

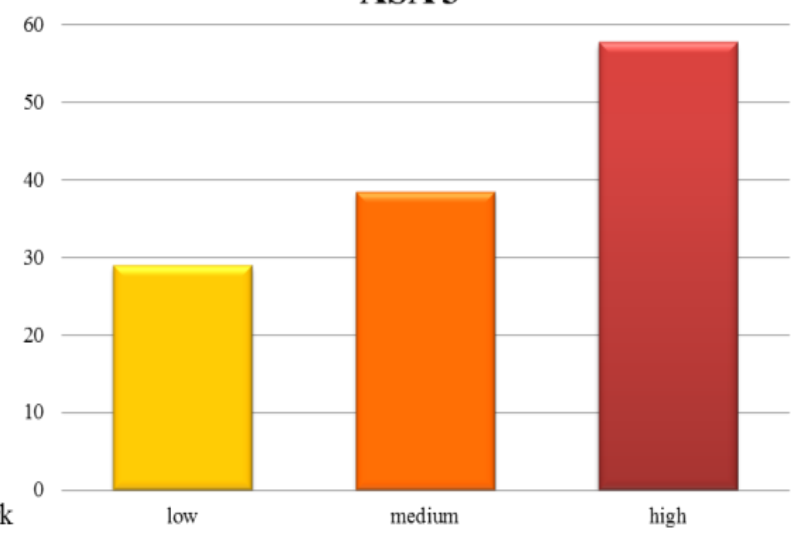

Figure 3: Patient segmentation by information deficiency and its impact on provider assessment time.

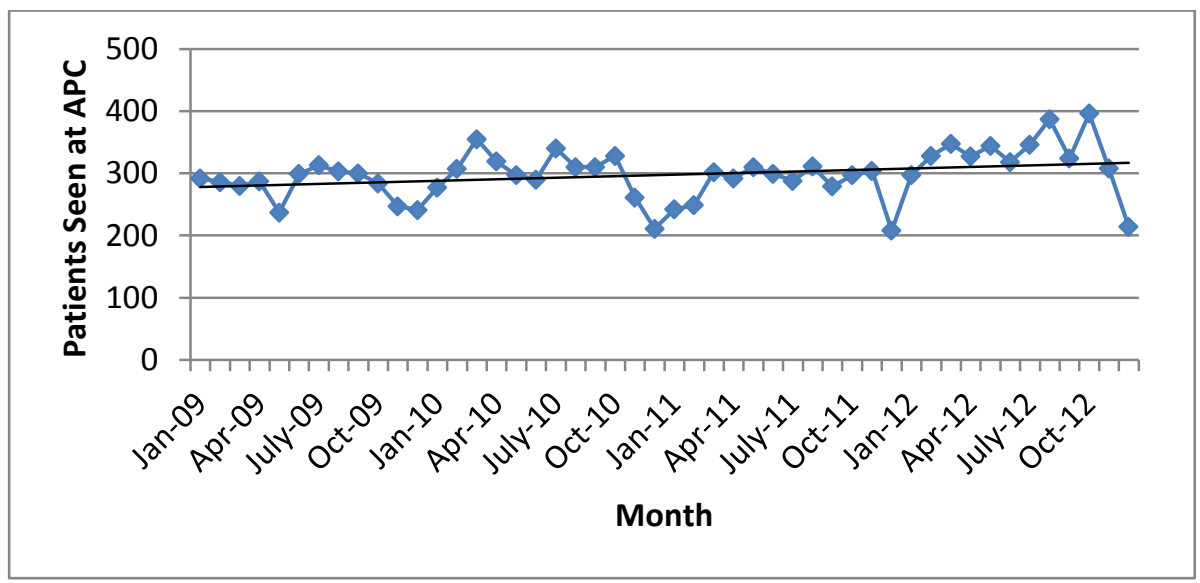

Figure 4: Monthly demand for APC service from 2009-2012.

Combined, these issues posed a major challenge for APC and the implementation of the PCSH model because the number of patients processed by APC was expected to more than double under the directive to screen all patients. We addressed this challenge using simulation.

\section{THE APC SIMULATION}

\subsection{The Simulation Model}

Using the observational and historical data along with the process mapping information described in the last section, we constructed a process simulation of APC using the Arena simulation software (Kelton et 
al. 2010). Figure 5 contains a screen capture of the simulation model. The simulation model captures the steps in the process: patient check-in and registration (patient arrivals and registration), a visit to the nurse for vitals and other information (nurse process), provider examination with faculty oversight (provider process), information gathering and data entry into the EMR after the patient is discharged (provider wrap-up), and patient discharge (end of process). The simulation also models the staffing and scheduling requirements for the resources (providers and nurse).

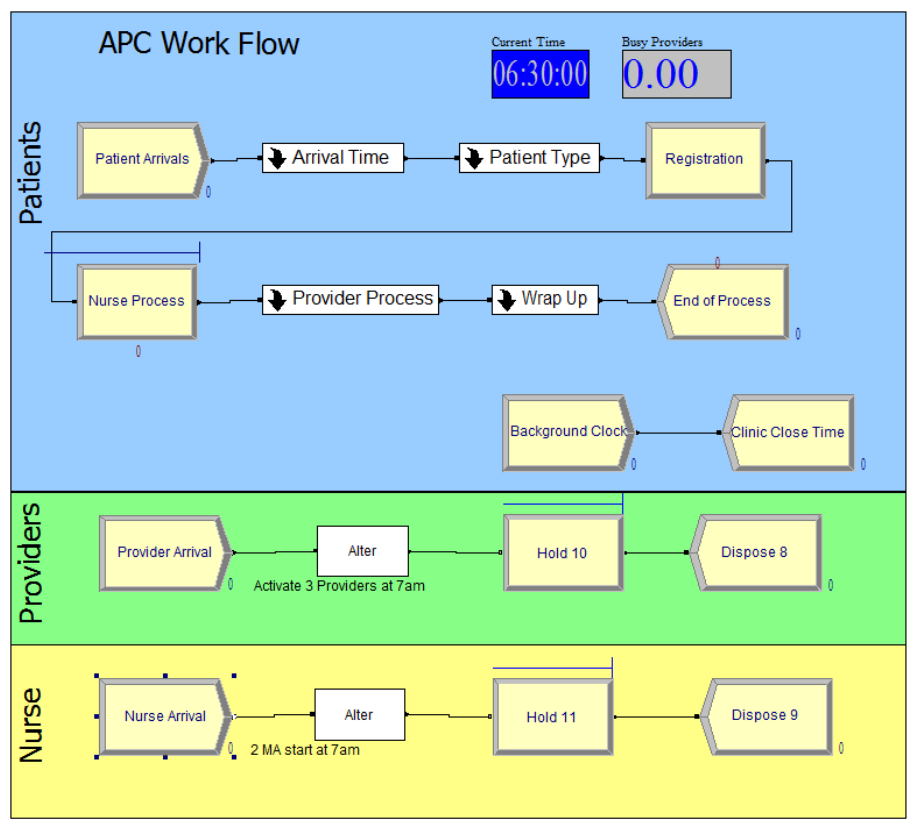

Figure 5: Arena simulation model of APC.

Models for patient arrivals and processing times in the simulation were estimated from the observational and historical data using the Arena Input Analyzer (Arena Simulation Software 2013), EasyFit (EasyFit 2013), StatTools (Palisade StatTools 2013) and STATA (STATA 2013). Table 1 provides a summary of these models. It is important to note that provider assessment (or provider process) times and provider wrap-up times are represented as functions of the information deficiency in the simulation model which is to be expected based on Figure 3. Provider assessment time was also found to be a function of the surgical complexity and the patient health complexity. Surgical complexity was assessed by the APC attending physician as low, medium, and high based on the complexity of the surgical procedure (as described in Eagle et al. 2002). Additionally, patient health complexity was assessed as low (patient in relatively good health), medium, and high (patient in relatively poor health). This assessment was guided by the American Society of Anesthesiologists (ASA) Physical Status Classification System (ASA 2013). These factors were also included in the simulation model.

Table 1: Models for patient arrivals and processing times in the APC simulation.

\begin{tabular}{|l|l|l|l|l|l|}
\hline $\begin{array}{c}\text { \# of Patients } \\
\text { Per Day }\end{array}$ & \multicolumn{1}{|c|}{$\begin{array}{c}\text { Arrival } \\
\text { Process }\end{array}$} & Registration & $\begin{array}{c}\text { Nurse } \\
\text { Screening }\end{array}$ & \multicolumn{1}{c|}{$\begin{array}{c}\text { Provider } \\
\text { Assessment }\end{array}$} & \multicolumn{1}{c|}{$\begin{array}{c}\text { Provider } \\
\text { Wrap-up }\end{array}$} \\
\hline $\begin{array}{l}\text { Discrete } \\
\text { Empirical }\end{array}$ & $\begin{array}{l}\text { Johnson SU } \\
\text { (deviation off } \\
\text { scheduled } \\
\text { times) }\end{array}$ & Lognormal & $\begin{array}{l}\text { Erlang }+ \\
\text { Triangular }\end{array}$ & $\begin{array}{l}\text { Regression (function } \\
\text { of surgical and patient } \\
\text { complexity, and in- } \\
\text { formation deficiency) }\end{array}$ & $\begin{array}{l}\text { Regression } \\
\text { (function of in- } \\
\text { formation defi- } \\
\text { ciency) }\end{array}$ \\
\hline
\end{tabular}


We found that patient registration at the clinic followed a Lognormal distribution. We used a Discrete Empirical distribution for the number of patients per day because no parameter distribution was found to provide a good fit to the data. An Erlang distribution was used for nurse screening. However, after the fact, we found "gaps" in the data in which the nurse was summoned to perform other duties. These gaps were mostly explained by requests for the nurse to conduct electrocardiogram (EKG) tests on the patients. Since there was only one nurse we were able glean some of these times from the data and model them using a Triangular distribution. We will describe the models used for the other three processes in more detail.

\subsubsection{Model for Patient Arrivals}

To model arrivals, we use the deviation time between arrival and scheduled times. Employing EasyFit we found that the Johnson SU performs the best among 36 candidate distributions in terms of Kolmogorov Smirnov, Anderson Darling and Chi-squared tests. We believe Johnson SU is appropriate for patient arrivals for two reasons: (1) it allows for heavier tails which correspond to extreme deviation values due to logistical challenges faced by the patient population (e.g., inner city transportation issues); and (2) it accommodates for a high peak at zero since many patients arrive punctually. A fitting comparison between Johnson SU and the Normal distribution (the other candidate distribution to which we gave serious consideration due to studies like Cayirli et al. 2006) using histogram/density function and P-P plots is shown in Figure 6. Incidentally, our findings are in line with Alexopoulos et al. (2008) and Rohleder et al. (2011).

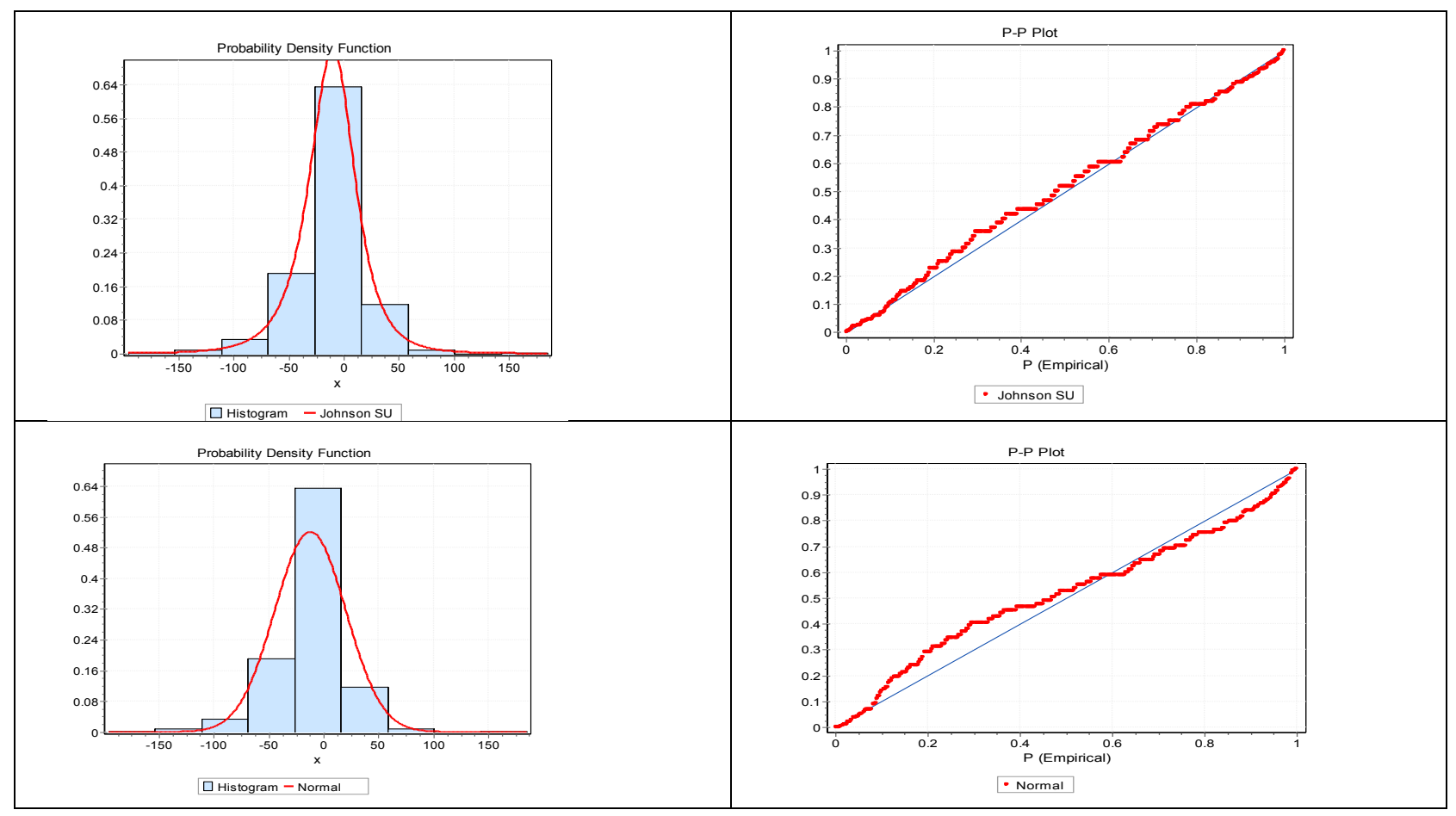

Figure 6. Arrival deviation fit in Johson SU and Normal distributions, generated by Easyfit. 


\subsubsection{Model for Provider Assessment}

For provider assessment time, we regressed the natural log of provider assessment time on dummy variables associated with surgical complexity (SurgeryLevel), patient complexity (PtLevel) and information deficiency (DataLevel) using backward regression analysis. The regression model is given in equation (1). The "low" level dummies were left out of the regression, and " $\mathrm{M}$ " and " $\mathrm{H}$ " refer to the high level dummy variables, respectively. The Adjusted $R^{2}=0.4044$ and the errors $\varepsilon \sim \operatorname{Normal}(0,0.353)$. All variables, except for SurgeryLevelH ( $\mathrm{p}$-value $=0.245)$, were statistically significant at the $5 \%$ level. DataLevelH has the largest coefficient in the regression model. It shows that high data deficiency plays a much greater role in longer provider assessment times than high surgical complexity and poor physical health. We elected to leave SurgeryLevelH in the regression model because it made practical and theoretical sense to do so. Also including this variable provided a slight improvement in the Adjusted $\mathrm{R}^{2}$ level. It is important to note that we did try including first-order interaction terms but the few that were found to be statistically significant in the model were collinear with the main effects and therefore not worth retaining.

Ln(Provider Assessment Time $)=$

$$
\begin{aligned}
& 3.06+0.37 *(\text { PtLevelH })+0.25 *(\text { PtLevelM })+0.10 *(\text { SurgeryLevelH })+0.11 * \\
& (\text { SurgeryLevelM })+0.56 *(\text { DataLevelH })+0.21 *(\text { DataLevelM })+\varepsilon
\end{aligned}
$$

We ran several diagnostic tests on the residuals using StatTools. Our choice of fitting residuals with a Normal distribution is supported by both a Q-Q Normal plot of the residuals (Figure 7) and the Lilliefors test which did not reject the null hypothesis of normality even at the $15 \%$ level. A residuals versus fitted plot (Figure 8) showed no evidence of non-linearity or heteroscedasticity.

\section{Q-Q Normal Plot of Residual}

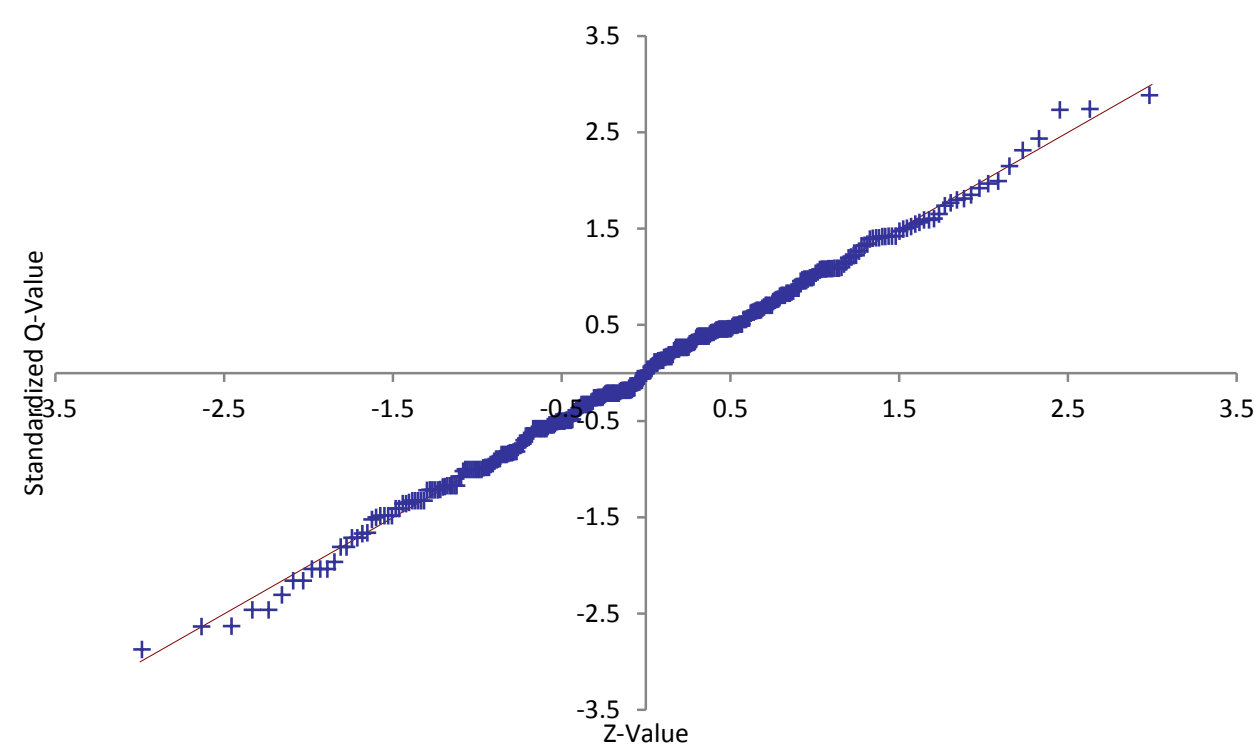

Figure 7: Q-Q Normal plot of the residuals by StatTools for the Provider Assessment Time regression. 
Morrice, Wang, Bard, Leykum, Noorily, and Veerapaneni

\section{Scatterplot of Residual vs Fitted}

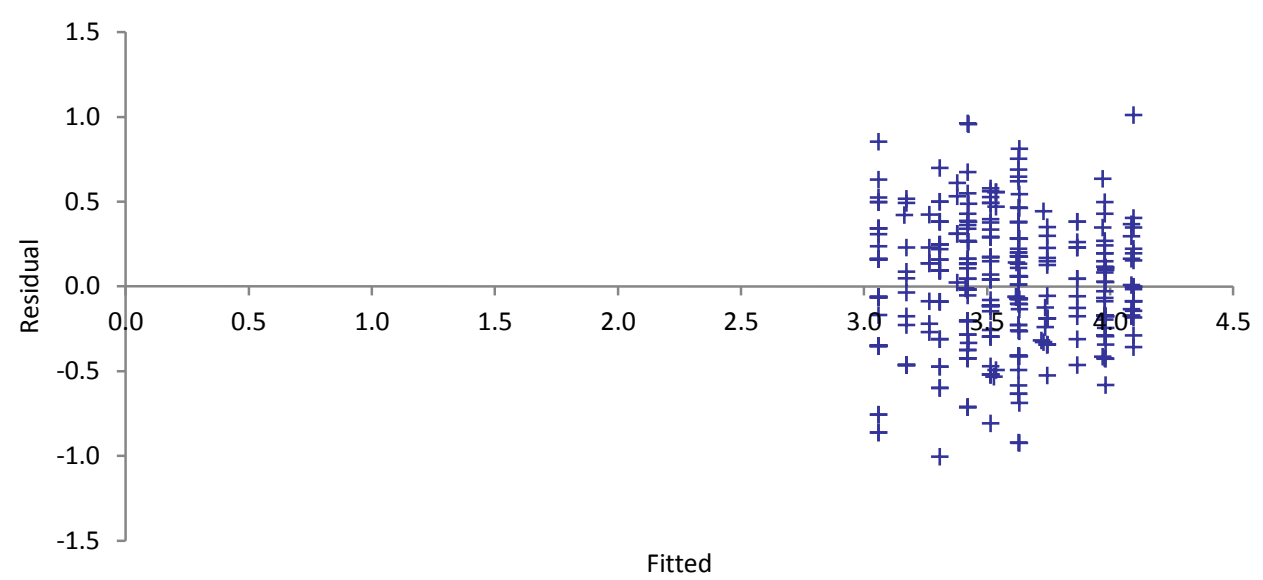

Figure 8. Residuals versus fitted value plot by StatTools for Provider Assessment Time regression.

\subsubsection{Model for Provider Wrap-up Times}

In the summer 2012 observational study of APC, we failed to collect data on the time taken by providers for additional information gathering and EMR data entry after the patient is discharged (provider wrapup). Consequently, we did not capture all the time a provider spent on each case and our simulation queuing statistics did not match the observed data (the simulation queue lengths were much shorter that what was being observed in the real system). Once we realized this, we conducted a focused observational study on provider wrap-up times and collected 107 observations on eight days in December 2012 through February 2013. The eight days were selected based on the availability of an observer to collect data, the convenience of the clinic, and to ensure that all days of the week were observed. Wrap-up times were regressed on SurgeryLevel, PtLevel and DataLevel. From backward regression, only DataLevelH and DataLevelM remained in the model (see equation 2). The Adjusted $\mathrm{R}^{2}$ for this model is 0.3053 . Again, the coefficients highlight the impact of information deficiency.

$$
\text { Wrap }- \text { up Time }=11.46+10.67 *(\text { DataLevelH })+5.13 *(\text { DataLevelM })+\varepsilon
$$

The residuals in the regression model in equation (2) did not satisfy the Normality assumption. Instead, a Beta distribution was found to fit the residuals using the Arena Input Analyzer.

\subsection{Model Validation}

Table 2 contains a comparison of the observed processing times from the summer 2012 data and the statistics generated by the simulation. The simulation was run for 600 days (roughly two years). Since the number of patient arrivals varied from one day to the next, 600 was chosen to ensure enough days were simulated even for the lowest probability number of patients per day. The results indicated that for most of the processes, the simulation is producing results that are not statistically distinguishable from the observed data. The one statistic of concern is the time for extra nurse duties. These data had to be crudely approximated from gaps in the data so this is not entirely surprising. We did not launch a separate study to get more data on the nurse for three reasons. First, the nurse is not the bottleneck in the system. Second, the nurse was recently replaced by two medical technicians (MAs) who perform roughly the same duties. This made what was formerly the nurse's duties even less likely to be a bottleneck since the capacity in- 
creased. Third, we are in the process of doing a more complete observational study and data collection of the MAs' performance. Hence, these data were not available at the time of the writing of this paper.

Table 2: Simulation validation statistics.

\begin{tabular}{|l|c|c|c|}
\hline \multicolumn{1}{|c|}{ Process } & Observational Statistics & \multicolumn{2}{c|}{ Simulated Statistics } \\
\hline Number of Patients per Day & Mean & Mean & 95\% CI Half-width \\
\hline Registration & 15.85 & 15.79 & 0.22 \\
\hline Nurse Queuing Time & 8.71 & 8.60 & 0.14 \\
\hline Nurse Screening Time & 20.67 & 21.39 & 0.64 \\
\hline Time for Extra Nurse Duties & 12.42 & 12.35 & 0.09 \\
\hline Provider Queuing Time & 5.57 & 2.7 & 0.01 \\
\hline Provider Assessment Time & 15.85 & 16.61 & 0.94 \\
\hline Provider Wrap-up Time & 38.42 & 38.22 & 0.37 \\
\hline Patient Waiting Time in System & 15.16 & 15.71 & 0.17 \\
\hline
\end{tabular}

\section{SIMULATION ANALYSIS FOR THE APC-COORDINATED PSCH MODEL}

Figure 9 illustrates key features of the APC-coordinated PSCH model. It relies on a screening tool that is completed by each patient at the referral clinics and a new $\mathrm{RN}$ who navigates each patient's case through the entire process. Using the results from the screening tool, the $\mathrm{RN}$ navigator confirms that appropriate tests are ordered and initiates retrieval of information for each patient. The $\mathrm{RN}$ navigator also determines whether a patient needs to make an APC visit or can be screened over the telephone prior to surgery. This decision is based on the overall health of the patient (sicker patients need to make a clinic visit, i.e., PtLevelM and PtLevelH patients). Phone screening can also be conducted by an RN, saving clinic provider time for those patients that really need to be seen in APC. The RN navigator completes the patient's file (or "passport") ensuring that the patient is ready for surgery.

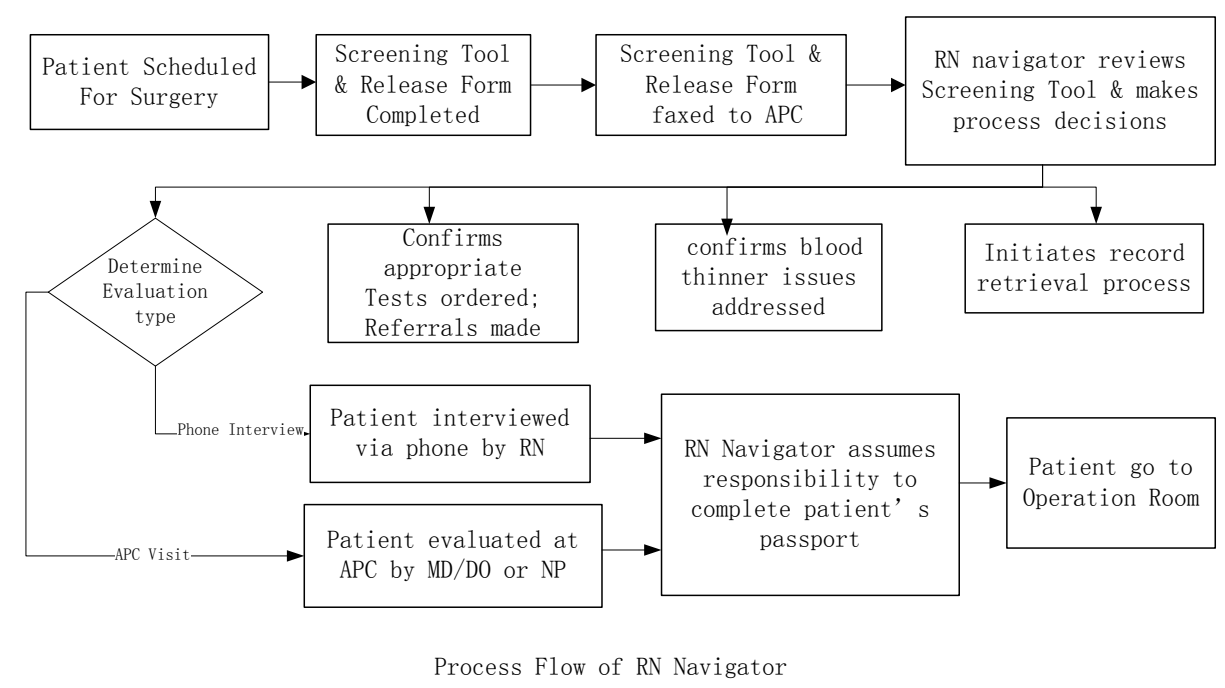

Figure 9: Key features of an APC-coordinated PCSH model. 
Based on an observational study conducted over a six-day period in February and March 2013 of all UHS's patients passing through Outpatient Surgery (221 patients), we determined that 25 patients/day represented the $97^{\text {th }}$ percentile of the number of patients needed to be seen in the clinic on any given day. Using the simulation, we determined if the screening tool and RN navigator were in place and all patients being seen in APC were low data deficiency patients (due to the screening tool and the upfront work of the RN navigator), then APC could handle up to 25 patients/day without any additional resources beyond the screening tool, the RN navigator, and perhaps an additional $\mathrm{RN}$ to assist on the telephone interviews.

Table 3 provides a summary of the results comparing a baseline simulation with all the current patient demographics against a simulation in which only medium and high complexity patients who have low data deficiencies are seen in the clinic (i.e., the APC-coordinated PCSH model). The results are based on 600 simulated days with 25 patients per day. Notice in the specifications all clinic staff report for duty at 7:00am when the clinic opens. While the first patients are scheduled at 7:30am, they may enter any time after 7:00am. This is designed to mimic reality where patients often show up early at the beginning of the day. In addition, two MAs are used instead of the nurse because the former has replaced the latter in APC going forward. Lastly, we estimated 21\% PtLevelH, 60\% PtLevelM, and 19\% PtLevelL patients from the summer 2012 observational study and used this in the baseline simulation. In the simulation of the APCcoordinated PCSH model, PtLevelL drops out, and PtLevelH and PtLevelM keep the same relative proportions.

Table 3: Comparison of baseline simulation and simulation of the APC-coordinated PCSH model.

\begin{tabular}{|c|c|c|c|c|}
\hline Case & \multicolumn{2}{|c|}{ Baseline } & \multicolumn{2}{|c|}{ PtLevelM, PtLevelH, and DataLevelL } \\
\hline Specifications & $\begin{array}{l}\text { First patient is sche } \\
\text { may enter APC sin } \\
2 \text { MAs start at } 7 \\
\text { start at } 7: 00 \mathrm{am} ; \\
21 \% \text { PtLevelH, } 60^{\circ} \\
21 \% \text { DataLevelH, }\end{array}$ & $\begin{array}{l}\text { duled at 7:30am but } \\
\text { e 7:00am; } \\
\text { 00am; } 3 \text { providers } \\
\text { PtLevelM; } \\
8 \% \text { DataLevelM. }\end{array}$ & $\begin{array}{l}\text { Same } \\
\text { Same } \\
\text { 25.9\% PtLevelH, } \\
\text { PtLevelL, 100\% D }\end{array}$ & $\begin{array}{l}\text { PtLevelM, no } \\
\text { elL. }\end{array}$ \\
\hline $\begin{array}{l}\text { Statistics (times } \\
\text { are in minutes) }\end{array}$ & Process Mean & Half-width & Process Mean & Half-width \\
\hline $\begin{array}{l}\text { Patient Total } \\
\text { Time in Clinic }\end{array}$ & 80.71 & $<1.14$ & 60.29 & $<0.47$ \\
\hline $\begin{array}{l}\text { Clinic Close } \\
\text { Time (after } \\
6: 30 \mathrm{am})\end{array}$ & $579.94(\sim 4: 10 \mathrm{pm})$ & 2.46 & $554.01(\sim 3: 44 \mathrm{pm})$ & 2.19 \\
\hline $\begin{array}{l}\text { Provider Utili- } \\
\text { zation }\end{array}$ & $82.41 \%$ & NA & $69.14 \%$ & NA \\
\hline $\begin{array}{l}\text { MA } \\
\text { Process Time }\end{array}$ & 12.43 & $<0.07$ & 12.41 & $<0.07$ \\
\hline $\begin{array}{l}\text { Provider } \\
\text { Assessment } \\
\text { Time }\end{array}$ & 38.36 & $<0.28$ & 31.99 & $<0.19$ \\
\hline $\begin{array}{l}\text { Provider Wrap } \\
\text { Up Time }\end{array}$ & 15.93 & $<0.13$ & 11.36 & $<0.11$ \\
\hline $\begin{array}{l}\text { Queuing Time } \\
\text { for MAs }\end{array}$ & 0.68 & $<0.04$ & 0.69 & $<0.04$ \\
\hline $\begin{array}{l}\text { Queuing Time } \\
\text { for Provider }\end{array}$ & 20.66 & $<0.93$ & 6.62 & $<0.32$ \\
\hline
\end{tabular}


Patient total time in clinic, clinic close time, the queuing statistics were most interesting to the clinic personnel and hospital administration. The elimination of medium and high data deficiency patients reduces provider assessment and wrap-up times significantly. Consequently the queuing time for the provider drops significantly. All these time savings result in a significant drop in the patient total time in the clinic. In addition, average clinic closing time drops significantly by about 25 minutes and provider utilization drops by about $13 \%$. All these improvement occur under a large per day patient load (at the $97^{\text {th }}$ percentile) of patients with more complex health issues. When these results were presented to top UHS hospital management, APC was given the go-ahead to implement the model in Figure 9. An RN has been added to the clinic staff to implement this process and pilot the screening tool.

\section{FUTURE WORK}

As the new PCSH model is developed, simulation will be used to forecast capacity and staffing level requirements in the new system, particularly for coordination between APC and the referral clinics, the new $\mathrm{RN}$ navigator, and the telephone interviews (see Figure 9). Additionally, plans are in the works to move APC to a new facility that will house both Anesthesiology and Internal Medicine services and further strengthen the concept of a PSCH at UHS. Again, we can use the simulation model to forecast capacity and staffing levels in the new system.

Another area for future work involves improved patient scheduling. There is a great deal of randomness in this system due to the variability associated with unbalanced patient arrivals, tardy patients, noshows, and last minute add-ons. We intend to explore more robust ways of patient scheduling to further improve APC operations.

\section{ACKNOWLEDGMENTS}

The authors would like to acknowledge and thank Ms. Carmen Sanchez and Mr. John Mark Atchley of UHS for providing data to support this project.

\section{REFERENCES}

Alexopoulos C. , D. Goldsman, J. Fontanesi, D. Kopald, J. R. Wilson. 2008. "Modeling Patient Arrivals in Community Clinics." Omega 36:33-43.

Arena Simulation Software. 2013. "Arena Simulation Software." Last accessed May 21, 2013. http://www.arenasimulation.com/Arena_Home.aspx.

The American Society of Anesthesiologists (ASA). 2011. "The Perioperiative or Surgical Home." Proposal to the ASA House of Delegates 2011 Session, August 21, 2011.

ASA. 2013. "The American Society of Anesthesiologists." Last accessed May 21, 2013. http://www.asahq.org/Home/For-Members/Clinical-Information/ASA-Physical-Status-ClassificationSystem.

Cayirli, T., E. Veral, H. Rosen. 2006. "Designing Appointment Scheduling Systems for Ambulatory Care Services." Healthcare Management Science 9: 47-58.

EasyFit. 2013. "Mathwave: Data Analysis and Simulation Software." Last accessed May 21, 2013. http://www.mathwave.com/products/easyfit.html.

Eagle K. A., P. B. Berger, H. Calkins. 2002. "ACC/AHA guideline update for perioperative cardiovascular evaluation for noncardiac surgery - executive summary. A report of the American College of Cardiology/American Heart Association Task Force on Practice Guidelines (Committee to update the 1996 guidelines on perioperative cardiovascular evaluation for noncardiac surgery)." Anesthesia \& Analgesia 94 (5): 1052-64.

Kelton, W.D., R.P. Sadowski, and N.B. Swets. 2010. Simulation with Arena, 5th ed., McGraw-Hill. 
Lahiri, A. and A. Seidmann. 2012. "Information Hang-Overs in Healthcare Service Systems." Manufacturing \& Service Operations Management 14(4): 634-653.

Palisade StatTools. 2013. "Palisade StatTools: Advance Statistical Analysis for Excel." Last accessed May 21, 2013. http://www.palisade.com/stattools/.

Rohleder, R. R., P. Lewkonia, D. P. Bischak, P. Duffy, R. Hendijani. 2011. "Using Simulation Modeling to Improve Patient Flow at an Outpatient Orthopedic Clinic." Health Care Management Science 14:135-145.

STATA. 2013. "STATA: Data Analysis and Statistical Software." Last accessed May 21, 2013. http://www.stata.com/.

\section{AUTHOR BIOGRAPHIES}

DOUGLAS J. MORRICE is a Professor of Supply Chain and Operations Management at The University of Texas at Austin. He is also Director of the University of Texas Supply Chain Management Center of Excellence. He has an ORIE Ph.D. from Cornell University. His research interests include simulation design, modeling, and analysis, healthcare delivery management, and supply chain risk management. Dr. Morrice was Co-Editor of the Proceedings of the 1996 Winter Simulation Conference, and 2003 Winter Simulation Conference Program Chair. He served as a representative for the INFORMS Simulation Society on the Winter Simulation Conference Board of Directors from 2004-2012. His email address is douglas.morrice@mccombs.utexas.edu.

DONGYANG (ESTER) WANG is a doctoral student of Supply Chain and Operations Management at The University of Texas at Austin. She received her B.S. in Industrial Engineering and Logistics Management from the Hong Kong University of Science and Technology. Her research interests are in the areas of healthcare management, simulation modeling, scheduling and supply chain management. Her email iswdy@utexas.edu.

JONATHAN F. BARD is a professor of Operations Research \& Industrial Engineering in the Mechanical Engineering Department at the University of Texas at Austin. He holds the Industrial Properties Corporation Endowed Faculty Fellowship and serves as the Associate Director of the Center for the Management of Operations and Logistics. He received a D.Sc. in Operations Research from The George Washington University, and has previously taught at the University of California - Berkeley and Northeastern University. His research interests are in the design and analysis of manufacturing systems, personnel scheduling, healthcare delivery, and algorithms for large-scale integer programs. He is a fellow of IIE and INFORMS, and a registered professional engineer in the State of Texas. His email address is jbard@mail.utexas.edu.

LUCI K. LEYKUM, M.D., M.B.A., M.Sc. is an Associate Professor of Medicine at the UT Health Science Center at San Antonio / South Texas Veterans Health Care System. Her interest is to reduce harm and improve outcomes for hospitalized patients. Her work has revolved around the application of complexity science to health care systems, using provider relationships and sense making to improve clinical outcomes. Her email address is Leykum@uthscsa.edu.

SUSAN H. NOORILY is a Clinical Professor of Anesthesiology at the University of Texas Health Science Center at San Antonio. She is also Medical Director of the Anesthesia Preoperative Clinic at the University Hospital. She received her B.S. and M.D. degrees at the University of Michigan where she also completed her residency training in Anesthesiology. Her research interests include perioperative medicine, preoperative evaluation, and preoperative laboratory testing. She was co-editor of the $3^{\text {rd }}$ and $4^{\text {th }}$ edi- 
Morrice, Wang, Bard, Leykum, Noorily, and Veerapaneni

tions of the textbook Decision Making in Anesthesiology (2000, 2007). Her e-mail address is noorily@uthscsa.edu.

POORNACHAND VEERAPANENI is a Statistician at the UT Health Science Center at San Antonio. He received his Masters in Clinical Investigation from University of Texas HSC San Antonio. His research interests are in the areas of healthcare information, statistics, and clinical research. His email address is Veerapaneni@uthscsa.edu. 